\title{
A Fokker-Planck model of the Boltzmann equation with correct Prandtl number for polyatomic gases
}

\author{
J. Mathiaud ${ }^{1}$, L. Mieussens ${ }^{2}$ \\ ${ }^{1} \mathrm{CEA}-\mathrm{CESTA}$ \\ 15 avenue des sablières - CS 60001 \\ 33116 Le Barp Cedex, France \\ (julien.mathiaud@cea.fr)
}

Fax number: (33)557045433

Phone number: (33)557046872

${ }^{2}$ Univ. Bordeaux, Bordeaux INP, CNRS, INRIA, IMB, UMR 5251, F-33400 Talence, France.

(Luc.Mieussens@math.u-bordeaux.fr)

\begin{abstract}
We propose an extension of the Fokker-Planck model of the Boltzmann equation to get a correct Prandtl number in the Compressible Navier-Stokes asymptotics for polyatomic gases. This is obtained by replacing the diffusion coefficient (which is the equilibrium temperature) by a non diagonal temperature tensor, like the Ellipsoidal-Statistical model is obtained from the Bathnagar-Gross-Krook model of the Boltzmann equation, and by adding a diffusion term for the internal energy. Our model is proved to satisfy the properties of conservation and a H-theorem. A Chapman-Enskog analysis shows how to compute the transport coefficients of our model. Some numerical tests are performed to illustrate that a correct Prandtl number can be obtained.
\end{abstract}

Keywords: Fokker-Planck model, Ellipsoidal-Statistical model,polyatomic gas, H-theorem, Prandtl number

\section{Introduction}

Numerical simulations of rarefied gas flows are fundamental tools to study the behavior of a gas in a system which length is of same order of magnitude as the mean free path of the gas molecules. For instance, these simulations are used in aerodynamics to estimate the heat flux at the wall of a re-entry space vehicle at high altitudes; numerical simulations are also used to estimate the attenuation of a micro-accelerometer by the surrounding gas in a micro-electro-mechanical system; a last example is when one wants to estimate the pumping speed or compression rate of a turbomolecular pump.

Depending on the problem, the flow can be in non-collisional, rarefied, or transitional regime, and several simulation methods exist. The most common method is the Direct Simulation Monte Carlo method (DSMC) proposed by Bird [3]: it is a stochastic method that simulate the flow with macro particles that mimic the real particles with transport and collisions. It works well for rarefied flows, but can be less efficient when the flow is close to continuous regime (even if there are other version of DSMC that are designed to work well in this regime: see for instance $[19,9])$. For non-collisional regimes, the Test Particle Monte Carlo (TPMC) method is very efficient and is a common tool for vacuum pumps [6]. For transition regimes, there exist mature deterministic solvers 
based on a direct approximation of the Boltzmann equation that can be used $[20,2,5,4,14,21]$. We refer to [18] or further references on these solvers. Among these solvers, some of them are based on model kinetic equations (BGK, ES-BGK, Shakhov): while these models keep the accuracy of the Boltzmann equation in the transition regime, they are much simpler to be discretized and give very efficient solvers $[16,5,20,4,2]$. These models simply replace the Boltzmann collision operator by a relaxation operator toward some equilibrium distribution, but still retain the elementary properties of the Boltzmann operator (collisional invariants, Maxwellian equilibrium). Two of them (BGK and ES-BGK) also satisfy the important entropy dissipation property (the so-called "H-theorem") [1].

Very recently, in a series of paper $[13,12,10,11]$, Jenny et al. proposed a very different and innovative approach: they proposed to use a different model equation known as the Fokker-Planck model to design a rarefied flow solver. In this model, the collisions are taken into account by a diffusion process in the velocity space. Like the model equations mentioned above, this equation also satisfy the main properties of the Boltzmann equation, even the H-theorem. Instead of using a direct discretization of this equation, the authors used the equivalent stochastic interpretation of this equation (the Langevin equations for the position and velocity of particles) that are discretized by a standard stochastic ordinary differential equation numerical scheme. This approach turned out to be very efficient, in particular in the transition regime, since it is shown to be insensitive to the number of simulated particles, as opposed to the standard DSMC. However, this FokkerPlanck model is parametrized by a single parameter, like the BGK model, and hence cannot give the correct transport coefficients in near equilibrium regimes: this is often stated by showing that the Prandtl number-which is the ratio between the viscosity to the heat transfer coefficient-has an incorrect value. The authors have proposed a modified model that allow to fit the correct value of the Prandtl number [12], and then have extended it to more complex flows (multi-species [10] and diatomic [11]). However, even if the results obtained with this approach seem very accurate, it is not clear that these models still satisfy the H-theorem.

In a previous paper [17], we have proposed an alternative modification of the Fokker-Planck equation for which we were able to prove the H-theorem and to obtain the correct Prandtl number. Our approach follows more or less the ideas used to transform the BGK model into the ES-BGK model, and our new model is called the ES-Fokker-Planck model (ES-FP), for monoatomic gases. In the present paper, we continue our work and extend our ES-FP model to polyatomic gases. To do so, we follow the approach used in [1] to derive a ES-BGK model for polyatomic gases. Again, we are able to prove that this model satisfies the $\mathrm{H}$-theorem and that it gives the correct transport coefficients in its hydrodynamic limit. We also show numerical experiments that confirm our analysis for some space homogenous problems.

The outline of this paper is the following. In section 2, our model and its properties are proved, including the H-theorem. A Chapman-Enskog analysis is made in section 3 to compute the transport coefficients obtained with this model. In section 4, we show that our model can include a variable parameter that allow to fit the correct Prandtl number and the viscosity parameter for polyatomic gases. Our numerical tests are shown in section 5, and conclusions and perspectives are presented in section 6 . 


\section{The Fokker-Planck model for polyatomic gases}

\subsection{The Boltzmann equation for polyatomic gases}

We consider a gas described by the mass density of particles $f(t, x, v, I)$ that at time $t$ have the position $x$, the velocity $v$ and an internal energy parameter $I$ (note that both position $x$ and velocity $v$ are vectors and that $I$ is a scalar). The internal energy of a particle is equal to $\varepsilon(I)=I^{\frac{2}{\delta}}, \delta$ being linked to the number of degrees of freedom of the polyatomic gas ( $\delta=2$ for diatomic gases). The corresponding macroscopic quantities are $(\rho, \rho u, E)=\left\langle\left(1, v,\left(\frac{1}{2}|v|^{2}\right)+\varepsilon(I)\right) f\right\rangle$, where $\rho, \rho u$, and $E$ are the mass, momentum, and energy densities, and $\langle\phi\rangle=\int_{\mathbb{R}^{3}} \phi(v, I) d v d I$ for any velocity dependent function. The temperature $T$ of the gas is defined by relation $E=\frac{1}{2} \rho|u|^{2}+\frac{3+\delta}{2} \rho R T$, where $R$ is the gas constant, and the pressure is $p=\rho R T$. We can also define a translational energy $E_{t r}$, a translationnal temperature $T_{t r}$, an internal energy $E_{\text {int }}$ and an internal energy temperature $T_{\text {int }}$ through:

$$
\begin{aligned}
& E_{t r}=\frac{3}{2} \rho R T_{t r}=\left\langle\frac{1}{2}|v-u|^{2} f\right\rangle \\
& E_{\text {int }}=\frac{\delta}{2} \rho R T_{\text {int }}=\langle\varepsilon(I) f\rangle,
\end{aligned}
$$

so that:

$$
T=\frac{3}{3+\delta} T_{t r}+\frac{\delta}{3+\delta} T_{i n t}
$$

The evolution of the gas can be governed by the following Boltzmann equation (if we do not take into account exchanges in internal energy)

$$
\partial_{t} f+v \cdot \nabla_{x} f=Q(f, f)
$$

where $Q(f, f)$ is for instance the Borgnakke-Larsen kernel of collision described in $([8,7])$ which allows reversible exchanges of energy between internal energy and translationnal energy of the particles.

It is well known that this operator conserves the mass, momentum, and energy, and that the local entropy $H(f)=\langle f \log f\rangle$ is locally non-increasing. This means that the effect of this operator is to make the distribution $f$ relax towards its own local Maxwellian distribution, which is defined by

$$
M_{p}(f)(v, I)=\frac{\rho \Lambda_{\delta}}{(2 \pi)^{3 / 2}(R T)^{(3+\delta) / 2}} \exp \left(-\frac{|v-u|^{2}}{2 R T}-\frac{I^{2 / \delta}}{R T}\right),
$$

where $\Lambda_{\delta}=\left(\int_{0}^{+\infty} \exp \left(-I^{2 / \delta}\right) d I\right)^{-1}$.

For the sequel, it is useful to define another macroscopic quantity, which is not conserved: the "temperature" tensor, defined by

$$
\Theta:=\frac{1}{\rho}\langle(v-u) \otimes(v-u) f\rangle .
$$

In an equilibrium state (that is to say when $f=M(f)$ ), $\Theta$ reduces to the isotropic tensor $R T I$. 


\subsection{The ES-Fokker-Planck model for polyatomic gases}

\subsubsection{Some facts about the ES-Fokker Planck model for monoatomic gases}

In [17] we extended the standard Fokker-Planck model for the Boltzmann equation by modifying its collision kernel $D(f)$ through:

$$
\begin{aligned}
& \partial_{t} f+v \cdot \nabla_{x} f=D(f)=\frac{1}{\tau} \nabla_{v} \cdot\left((v-u) f+\Pi \nabla_{v} f\right), \\
& \Pi=(1-\nu) R T I d+\nu \Theta .
\end{aligned}
$$

with $\tau$ a characteristic relaxation time and $\nu$ a parameter allowing to recover the correct Prandtl number for monoatomic gases when $\nu=-\frac{1}{2}$. Now we remind the results proved in [17].

Proposition 2.1. The tensor $\Pi$ is symmetric positive definite for every tensor $\Theta$ if, and only if,

$$
-\frac{R T}{\lambda_{\max }-R T}<\nu<\frac{R T}{R T-\lambda_{\min }}
$$

where $\lambda_{\max }$ and $\lambda_{\min }$ are the (positive) maximum and minimum eigenvalues of $\Theta$. Moreover $\Pi$ is positive definite independently of $\Theta$ as long as: $-\frac{1}{2}<\nu<1$.

Proposition 2.2. The operator D satisfies:

$$
D(f)=\frac{1}{\tau} \nabla_{v} \cdot\left(\Pi G(f) \nabla_{v} \frac{f}{G(f)}\right)=\frac{1}{\tau} \nabla_{v} \cdot\left(\Pi f \nabla_{v} \log \left(\frac{f}{G(f)}\right)\right),
$$

where $G(f)$ is the anisotropic Gaussian defined by

$$
G(f)=\frac{\rho}{\sqrt{\operatorname{det}(2 \pi \Pi)}} \exp \left(-\frac{(v-u) \Pi^{-1}(v-u)}{2}\right),
$$

which has the same 5 first moments as $f$ and $\Pi$ for temperature tensor:

$$
\left\langle\left(1, v, \frac{1}{2}|v|^{2}\right) G(f)\right\rangle=(\rho, \rho u, E), \quad\langle(v-u) \otimes(v-u) G(f)\rangle=\rho \Pi .
$$

If $\nu$ satisfies (8) and $\nu<1$, it conserves mass, momentum, energy and entropy is dissipated:

$$
\left\langle\left(1, v, \frac{1}{2}|v|^{2}\right) D(f)\right\rangle=0,\langle D(f) \log f\rangle \leq 0,
$$

Moreover as expected the equilibrium property holds:

$$
D(f)=0 \Leftrightarrow f=G(f) \Leftrightarrow f=M(f) .
$$

\subsubsection{The ES-Fokker-Planck model for polyatomic gases}

Our extension of the previous model to polyatomic gases is based on two ideas. First, the variation of the internal energy of the particles due to collisions are taken into account by an additional diffusion term (with respect to the internal energy variable) in the collision operator. Then, the transfers between translational and internal energies are taken into account by an additional parameter $\theta$, 
like in the construction of the ESBGK model for polyatomic gases proposed in [1]. This leads to our ES-Fokker-Planck model for polyatomic gases:

$$
\partial_{t} f+v \cdot \nabla_{x} f=D(f)
$$

where the collision operator is defined by

$$
D(f)=\frac{1}{\tau}\left(\nabla_{v} \cdot\left((v-u) f+\Pi \nabla_{v} f\right)+\partial_{I}\left(\delta f I+\frac{\delta^{2}}{2} R T_{r e l} I^{2-\frac{2}{\delta}} \partial_{I} f\right)\right),
$$

where $\tau$ is the relaxation time, and $\Pi$ is the following combination between the temperature tensor $\Theta$, the translational temperature tensor $R T_{t r} I d$ and its equilibrium value $R T I d$ :

$$
\Pi=(1-\theta)\left((1-\nu) R T_{t r} I d+\nu \Theta\right)+\theta R T I d,
$$

where the coefficients $\nu$ and $\theta$ are some free parameters, and the temperature $T_{r e l}$ is defined by

$$
T_{r e l}=(1-\theta) T_{i n t}+\theta T \text {. }
$$

This model is the natural extension of the monoatomic ESFP model to get the polyatomic anisotropic Gaussian as equilibrium for polyatomic gases, as it was done for the ESBGK model for polyatyomic gases (see [1]).

Now, we give a few comments about parameters $\theta$ and $\delta$. First, it can be seen that $\theta$ governs the reversible exchanges between internal and translational energies. Indeed, for a space homogeneous problem (we neglect the space dependence of the problem), it can easily be seen that the rate at which the energy is transferred from internal energy to translational energy is $\frac{3+\delta}{2 \delta \theta} \tau E_{\text {int }}$. The corresponding relaxation time then is $\frac{3+\delta}{2 \delta \theta} \tau$, and a relaxation collision number on the collision rate time-scale $\tau$ (see [3]) can be defined by $Z=\frac{3+\delta}{2 \delta \theta}$ which is clearly parametrized by $\theta$. Consequently, it is clear that when $\theta=0$ we no longer have exchanges between internal and translational energies. Note that this model is compatible with the monoatomic model: indeed, when $\delta=0$ we immediatly recover the monoatomic model since in this case $T_{t r}=T$.

For the following, it is usefull to rewrite model (12) a bit differently. We define the generalized tensor $\Omega$ by

$$
\Omega=\left(\begin{array}{c|c}
\Pi & 0 \\
\hline 0 & \frac{\delta^{2}}{2} R T_{r e l} I^{2-\frac{2}{\delta}}
\end{array}\right),
$$

so that the collision operator of (12) reads

$$
D(f)=\frac{1}{\tau} \nabla_{v, I} \cdot\left(\left(\begin{array}{c}
v-u \\
\delta I
\end{array}\right) f+\Omega \nabla_{v, I} f\right)
$$

where $\nabla_{v, I}$ denotes the derivative operator with respect to both $v$ and $I$ variables. We can now define the generalized Gaussian $G_{p}(f)$ for polyatomic gases as:

$$
G_{p}(f)=\frac{\rho \Lambda_{\delta}}{\sqrt{\operatorname{det}(2 \pi \Pi)\left(R T_{r e l}\right)^{\delta / 2}}} \exp \left(-\frac{1}{2}\left(\begin{array}{c}
v-u \\
\delta I
\end{array}\right)^{T} \Omega^{-1}\left(\begin{array}{c}
v-u \\
\delta I
\end{array}\right)\right) .
$$


One can note that contrary to the case of monoatomic gases the tensor $\Omega$ depends on the variables of the phase space through the internal parameter. The generalized Maxwellian $M_{p}(f)$ of equilibrium for polyatomic gases (5) can also be defined as:

$$
M_{p}(f)=\frac{\rho \Lambda_{\delta}}{(2 \pi)^{3 / 2}(R T)^{\frac{3+\delta}{2}}} \exp \left(-\left(\begin{array}{c}
\frac{v-u}{\sqrt{2 R T}} \\
\frac{I^{1 / \delta}}{\sqrt{R T}}
\end{array}\right)^{T}\left(\begin{array}{c}
\frac{v-u}{\sqrt{2 R T}} \\
\frac{I^{1 / \delta}}{\sqrt{R T}}
\end{array}\right)\right)
$$

We now look for the properties of the collision kernel $D(f)$ as it was done in the monoatomic case.

Proposition 2.3. The operator $D$ has two other equivalent formulations:

$$
D(f)=\frac{1}{\tau} \nabla_{v, I} \cdot\left(\Omega G_{p}(f) \nabla_{v, I} \frac{f}{G_{p}(f)}\right),
$$

and

$$
D(f)=\frac{1}{\tau} \nabla_{v, I} \cdot\left(\Omega f \nabla_{v, I} \log \left(\frac{f}{G_{p}(f)}\right)\right),
$$

Moreover $G_{p}$ has the same 5 first moments as $f$

$$
\left\langle\left(1, v, \frac{1}{2}|v|^{2}+I^{2 / \delta}\right) G_{p}(f)\right\rangle=(\rho, \rho u, \rho E),
$$

and has its translationnal temperature tensor equal to $\left\langle(v-u) \otimes(v-u) G_{p}(f)\right\rangle / \rho=\Pi$.

Proof. Relations (19) and (20) are simple consequences of the relations $\nabla_{v} G_{p}(f)=-\Pi^{-1}(v-$ $u) G_{p}(f)$ and $\partial_{I} G_{p}(f)=-\frac{2 I^{2 / \delta-1}}{\delta R T_{r e l}} G_{p}(f)$. The moments of $G_{p}(f)$ are given by standard Gaussian integrals.

Proposition 2.4 (Condition of definite positiveness of $\Pi$ ).

Assume $0<\theta<1$, the tensor $\Pi$ is symmetric positive definite if, and only if,

$$
-\frac{R T_{t r}+\frac{\theta}{1-\theta} R T}{\lambda_{\max }-R T_{t r}}<\nu<\frac{R T_{t r}+\frac{\theta}{1-\theta} R T}{R T_{t r}-\lambda_{\min }}
$$

for every tensor $\Theta$ ( $\lambda_{\max }$ and $\lambda_{\min }$ being its (positive) maximum and minimum eigenvalues). Moreover $\Pi$ is positive definite independently of the eigenvalues of $\Theta$ as long as:

$$
-\frac{1}{2}<\nu<1
$$

Proof. Since $\Theta$ is symmetric and positive definite, it is diagonalizable and its eigenvalues are positive. Moreover, because of the definition of $\Pi$, both $\Theta$ and $\Pi$ are diagonalizable in the same orthogonal basis. Then there exists an invertible orthogonal matrix $P$ such that

$$
P \Theta P^{T}=\left(\begin{array}{ccc}
\lambda_{1} & 0 & 0 \\
0 & \lambda_{2} & 0 \\
0 & 0 & \lambda_{3}
\end{array}\right)
$$


and

$$
P \Pi P^{T}=\left(\begin{array}{ccc}
\gamma_{1} & 0 & 0 \\
0 & \gamma_{2} & 0 \\
0 & 0 & \gamma_{3}
\end{array}\right) .
$$

with $\gamma_{i}=(1-\theta)\left((1-\nu) R T_{t r}+\nu \lambda_{i}\right)+\theta R T$. Clearly $\Pi$ is positive definite if, and only if, $\gamma_{i}>0$ for every $i$, which leads to:

$$
-\frac{R T_{t r}+\frac{\theta}{1-\theta} R T}{\lambda_{\max }-R T_{t r}}<\nu<\frac{R T_{t r}+\frac{\theta}{1-\theta} R T}{R T_{t r}-\lambda_{\min }},
$$

which is (21).

Note that $R T_{t r}=\operatorname{trace}(\Theta) / 3$ is the mean value of the eigenvalues $\lambda_{i}$ of $\Theta$. Consequently, when $\lambda_{\max }$ gets close to the $R T_{t r}$, then $\lambda_{\min }$ gets close to $R T_{t r}$ too-which happends when $f$ is close to a Maxwellian equilibrium-and hence our analysis shows that all the $\gamma_{i}$ are positive, indendently of $\nu$. This is consistent with (21) wich shows that $\nu$ can take any value.

The sufficient condition(22) is obtained as follows. At worse $\lambda_{\min }$ is equal to zero and $\theta$ is equal to 0 so that

$$
\nu<\frac{R T_{t r}}{R T_{t r}-0}=1
$$

At worse $\lambda_{\max }$ is equal to $\operatorname{trace}(\Theta)=3 R T_{t r}$ and $\theta$ is equal to 0 so that

$$
\nu>-\frac{R T_{t r}}{3 R T_{t r}-R T_{t r}}=-\frac{1}{2} .
$$

Now, we state that $D$ has the same conservation and entropy properties as the Boltzmann collision operator $Q$.

Proposition 2.5. We assume $\nu$ satisfies (21) and that $\nu<1$. The operator $D$ conserves the mass, momentum, and energy:

$$
\left\langle\left(1, v, \frac{1}{2}|v|^{2}+I^{2 / \delta}\right) D(f)\right\rangle=0
$$

it satisfies the dissipation of the entropy:

$$
\langle D(f) \log f\rangle \leq 0
$$

and we have the equilibrium property:

$$
D(f)=0 \Leftrightarrow f=G_{p}(f) \Leftrightarrow f=M_{p}(f) .
$$

Proof of the conservation property. We take any function $\phi(v)$ and by integration by parts, we get

$$
\langle D(f) \phi\rangle=-\frac{1}{\tau}\left\langle\left((v-u) f+\Pi \nabla_{v} f\right) \cdot \nabla_{v} \phi-\left(\delta f I+\frac{\delta^{2}}{2} R T_{r e l} I^{2-\frac{2}{\delta}} \partial_{I} f\right) \partial_{I} \phi\right\rangle .
$$

With $\phi(v)=1$, we directly get $\langle D(f)\rangle=0$. With $\phi(v)=v_{i}$, we get

$$
\left\langle D(f) v_{i}\right\rangle=-\frac{1}{\tau}\left\langle\left(v_{i}-u_{i}\right) f+\Pi_{i, j} \partial_{v_{j}} f\right\rangle=0,
$$


since $\left\langle v_{i} f\right\rangle=\rho u_{i}=\rho\left\langle v_{i} f\right\rangle$ and $\left\langle\partial_{v_{j}} f\right\rangle=0$. For $\phi(v, I)=\frac{1}{2}|v|^{2}+I^{2 / \delta}$, we get

$$
\begin{aligned}
\left\langle D(f)\left(\frac{1}{2}|v|^{2}+I^{2 / \delta}\right)\right\rangle & =-\frac{1}{\tau}\left\langle\left(\left(v_{i}-u_{i}\right) f+\Pi_{i, j} \partial_{v_{j}} f\right) v_{i}-\left(\delta f I+\frac{\delta^{2}}{2} R T_{r e l} I^{2-\frac{2}{\delta}} \partial_{I} f\right) \frac{2}{\delta} I^{\frac{2}{\delta}-1}\right\rangle \\
& =-\frac{1}{\tau}\left(3 \rho R T_{t r}-\rho \operatorname{trace}(\Pi)\right)-\frac{1}{\tau}\left(\rho \delta R T_{\text {int }}-\rho \delta R T_{r e l}\right) .
\end{aligned}
$$

We note that $(14)$ implies trace $(\Pi)=(1-\theta)\left(3 R T_{t r}\right)+\theta(3 R T)$. Then we use the definitions $(3)$ and (15) of $T$ and $T_{r e l}$ to obtain that the last integral is zero.

Proof of the entropy property. This proof, which rather long, is based on two main ideas. First, as noticed in the proof of proposition 2.4, $\Theta$ and $\Pi$ are symmetric and can be diagonalized in the same orthogonal basis. Like it is done in [17], we will now suppose that we work in this basis so that:

$$
\Theta=\left(\begin{array}{ccc}
\lambda_{1} & 0 & 0 \\
0 & \lambda_{2} & 0 \\
0 & 0 & \lambda_{3}
\end{array}\right),
$$

and

$$
\Pi=\left(\begin{array}{ccc}
\gamma_{1} & 0 & 0 \\
0 & \gamma_{2} & 0 \\
0 & 0 & \gamma_{3}
\end{array}\right) .
$$

with $\gamma_{i}=(1-\theta)\left((1-\nu) R T_{t r}+\nu \lambda_{i}\right)+\theta R T$. Moreover, we will make use of the well known Jensen inequality: for every convex function $x \mapsto C(x)$ and every mean $\mu$, we have $C\left(\mu\left(a_{1}, a_{2}, \ldots, a_{n}\right)\right) \leq$ $\mu\left(C\left(a_{1}\right), C\left(a_{2}\right), \ldots, C\left(a_{n}\right)\right)$, for every real $a_{1}, a_{2}, \ldots, a_{n}$, with equality if, and only if, all the $a_{i}$ are equal or $C$ is linear. This inequality will be used twice. First, since $\sum_{i=1}^{3} \lambda_{i}=\operatorname{trace}(\Theta)=$ $3 R T_{t r}$, we have $R T_{t r}=\frac{1}{3} \sum_{i=1}^{3} \lambda_{i}=\mu\left(\lambda_{1}, \lambda_{2}, \lambda_{3}\right)$, where $\mu$ is the arithmetic mean of the $\lambda_{i}$. Consequently, since the function $C(x)=1 / x$ is convex, the Jensen inequality induces $C\left(T_{T r}\right) \leq$ $\mu\left(C\left(\lambda_{1}\right), C\left(\lambda_{2}\right), C\left(\lambda_{3}\right)\right)$, which reads

$$
\frac{1}{R T_{t r}} \leq \frac{1}{3} \sum_{i=1}^{3} \frac{1}{\lambda_{i}} .
$$

Second, the definition (3) of the equilibrium temperature reads $T=\mu\left(T_{t r}, T_{\text {int }}\right)$, where $\mu(a, b)=$ $(3 a+\delta b) /(3+\delta)$ is a weighted average. Consequently, the Jensen inequality implies $C(T) \leq$ $\mu\left(C\left(T_{t r}\right), C\left(T_{\text {int }}\right)\right)$, which reads

$$
\frac{1}{T} \leq \frac{1}{3+\delta}\left(\frac{3}{T_{t r}}+\frac{\delta}{T_{i n t}}\right) .
$$


Now we compute $\langle D(f) \log f\rangle$ with an integration by parts to get

$$
\begin{aligned}
\langle D(f) \log (f)\rangle= & -\frac{1}{\tau}\left\langle\sum_{i=1}^{3}\left(\left(v_{i}-u_{i}\right) f+\gamma_{i} \partial_{v_{i}} f\right)\left(\frac{\partial_{v_{i}} f}{f}\right)-\left(\delta f I+\frac{\delta^{2}}{2} R T_{r e l} I^{2-\frac{2}{\delta}} \partial_{I} f\right)\left(\frac{\partial_{I} f}{f}\right)\right\rangle \\
= & -\frac{1}{\tau}\left\langle\sum_{i=1}^{3} \frac{\gamma_{i}}{\lambda_{i}}\left(\left(v_{i}-u_{i}\right) f+\lambda_{i} \partial_{v_{i}} f\right)\left(\frac{\partial_{v_{i}} f}{f}\right)+\sum_{i=1}^{3}\left(1-\frac{\gamma_{i}}{\lambda_{i}}\right) \partial_{v_{i}} f\left(v_{i}-u_{i}\right)\right\rangle \\
& -\frac{1}{\tau}\left\langle\left(\delta f I+\frac{\delta^{2}}{2} R T_{r e l} I^{2-\frac{2}{\delta}} \partial_{I} f\right)\left(\frac{\partial_{I} f}{f}\right)\right\rangle \\
= & -\frac{1}{\tau}\left\langle\sum_{i=1}^{3} \frac{\gamma_{i}}{\lambda_{i}}\left(\left(v_{i}-u_{i}\right) f+\lambda_{i} \partial_{v_{i}} f\right)\left(\frac{\partial_{v_{i}} f}{f}+\frac{\left(v_{i}-u_{i}\right)}{\lambda_{i}}-\frac{\left(v_{i}-u_{i}\right)}{\lambda_{i}}\right)\right\rangle \\
& +\frac{1}{\tau} \sum_{i=1}^{3}\left(1-\frac{\gamma_{i}}{\lambda_{i}}\right)\langle f\rangle \\
& -\frac{1}{\tau}\left\langle\left(\delta f I+\frac{\delta^{2}}{2} R T_{r e l} I^{2-\frac{2}{\delta}} \partial_{I} f\right)\left(\frac{\partial_{I} f}{f}\right)\right\rangle \\
= & -\frac{1}{\tau}\left\langle\sum_{i=1}^{3} \frac{\gamma_{i}}{\lambda_{i}} \frac{1}{f \lambda_{i}}\left(\left(v_{i}-u_{i}\right) f+\lambda_{i} \partial_{v_{i}} f\right)^{2}\right\rangle \\
& +\frac{1}{\tau}\left\langle\sum_{i=1}^{3} \frac{\gamma_{i}}{\lambda_{i}}\left(\left(v_{i}-u_{i}\right) f+\lambda_{i} \partial_{v_{i}} f\right)\left(\frac{\left(v_{i}-u_{i}\right)}{\lambda_{i}}\right)\right\rangle \\
& +\frac{1}{\tau} \sum_{i=1}^{3}\left(1-\frac{\gamma_{i}}{\lambda_{i}}\right)\langle f\rangle \\
& -\frac{1}{\tau}\left\langle\left(\delta f I+\frac{\delta^{2}}{2} R T_{r e l} I^{2-\frac{2}{\delta}} \partial_{I} f\right)\left(\frac{\partial_{I} f}{f}\right)\right\rangle \\
= & (A)+(B)+(C)+(D)
\end{aligned}
$$

The first term $(A)$ is clearly non positive. By using another integration by parts and the definition of the $\lambda_{i}$, it is easy to see that $(B)=0$. For $(C)$, we use the definition of $\gamma_{i}$ to find

$$
(C)=\frac{1}{\tau} \sum_{i=1}^{3}\left(1-\frac{(1-\theta)\left((1-\nu) R T_{t r}+\nu \lambda_{i}\right)+\theta R T}{\lambda_{i}}\right)\langle f\rangle .
$$

Then we expand the right-hand side

$$
(C)=\frac{3}{\tau}\left(1-(1-\theta) \nu-\left((1-\theta)(1-\nu) R T_{t r}+\theta R T\right) \frac{1}{3} \sum_{i} \frac{1}{\lambda_{i}}\right),
$$

and we use inequality (24) to find

$$
(C) \leq \frac{3 \theta}{\tau}\left(1-\frac{T}{T_{t r}}\right)\langle f\rangle
$$


For $(D)$, we try again to make appear a non positive term. We find

$$
\begin{aligned}
(D)= & -\frac{1}{\tau}\left\langle\left(\delta f I+\frac{\delta^{2}}{2} R T_{\text {rel }} I^{2-\frac{2}{\delta}} \partial_{I} f\right)\left(\frac{\partial_{I} f}{f}\right)\right\rangle \\
= & -\frac{1}{\tau} \frac{T_{r e l}}{T_{i n t}}\left\langle\left(\delta f I+\frac{\delta^{2}}{2} R T_{i n t} I^{2-\frac{2}{\delta}} \partial_{I} f\right)\left(\frac{\partial_{I} f}{f}+\frac{2}{\delta} \frac{I^{\frac{2}{\delta}-1}}{R T_{i n t}}-\frac{2}{\delta} \frac{I^{\frac{2}{\delta}-1}}{R T_{i n t}}\right)\right\rangle \\
& -\frac{1}{\tau}\left(1-\frac{T_{r e l}}{T_{i n t}}\right) \delta\left\langle I \partial_{I} f\right\rangle \\
= & -\frac{1}{\tau} \frac{T_{r e l}}{T_{i n t}}\left\langle\frac{1}{f \frac{\delta^{2}}{2} R T_{i n t} I^{2-\frac{2}{\delta}}}\left(\delta f I+\frac{\delta^{2}}{2} R T_{i n t} I^{2-\frac{2}{\delta}} \partial_{I} f\right)^{2}\right\rangle \\
& +\frac{1}{\tau} \frac{T_{r e l}}{T_{i n t}}\left\langle\left(\delta f I+\frac{\delta^{2}}{2} R T_{i n t} I^{2-\frac{2}{\delta}} \partial_{I} f\right)\left(\frac{2}{\delta} \frac{I^{\frac{2}{\delta}-1}}{R T_{i n t}}\right)\right\rangle \\
& +\frac{1}{\tau}\left(1-\frac{T_{r e l}}{T_{i n t}}\right) \delta\langle f\rangle \\
= & (D 1)+(D 2)+(D 3)
\end{aligned}
$$

Then $(D 1)$ is clearly non positive. For $(D 2)$, an integration by parts and the definition (2) of $T_{\text {int }}$ implies $(D 2)=0$. This shows that $(D) \leq(D 3)=\frac{1}{\tau}\left(1-\frac{T_{\text {rel }}}{T_{\text {int }}}\right) \delta\langle f\rangle$.

Finally, we collect all the inequalities obtained for $(A),(B),(C)$, and $(D)$ to find

$$
\begin{aligned}
\langle D(f) \log (f)\rangle & \leq \frac{3 \theta}{\tau}\left(1-\frac{T}{T_{t r}}\right)\langle f\rangle+\frac{\theta}{\tau} \delta\left(1-\frac{T}{T_{\text {int }}}\right)\langle f\rangle \\
& =\frac{(3+\delta) \theta}{\tau}\langle f\rangle\left(1-T \frac{1}{3+\delta}\left(\frac{3}{T_{t r}}+\frac{\delta}{T_{\text {int }}}\right)\right) \\
& \leq 0,
\end{aligned}
$$

from the Jensen inequality (25).

Proof of the equilibrium property. First, we assume that $f=M_{p}(f)$, as defined by (5). Then it can easily be seen that (1) and (2) imply $T_{t r}=T_{i n t}=T$, while (6) implies $\Theta=R T I d$. Using (14) and (15) and the previous relations yields $T_{r e l}=T$ and $\Pi=R T I d$. This shows that $G_{p}(f)$, as defined by (17), satisfies $G_{p}(f)=M_{p}(f)$, and hence $f=G_{p}(f)$. Finally, the formulation (19) of the collision operator implies $D(f)=0$.

Now, we assume that $D(f)=0$, and we want to prove that $f=M_{p}(f)$. This is where the entropy inequality is used: indeed, $D(f)=0$ implies $\langle D(f) \log f\rangle=0$, which is used as follows. First, this relation with inequality (29) imply $\frac{1}{3+\delta}\left(\frac{3}{T_{t r}}+\frac{\delta}{T_{i n t}}\right) \leq \frac{1}{T}$. This means that the Jensen inequality (25) is in fact an equality, and hence $T_{t r}=T_{i n t}=T$. Now, we remind that $\langle D(f) \log f\rangle=(A)+((C)+(D 3))+(D 1)$, where $(A),((C)+(D 3))$, and $(D 1)$ are non positive (see the proof of the entropy inequality). Then $\langle D(f) \log f\rangle=0$ implies in particular $(A)=0$, $(C)+(D 3)=0$ and $(D 1)=0$. Note that $(A)=0$ and $(D 1)=0$ imply

$$
\frac{\partial_{v_{i}} f}{f}+\frac{\left(v_{i}-u_{i}\right)}{R T}=0 \quad \text { and } \quad \frac{\partial_{I} f}{f}+\frac{2}{\delta} \frac{I^{\frac{2}{\delta}-1}}{R T}=0 .
$$


This shows that $f=\alpha \exp \left(-\sum_{i} \frac{\left(v_{i}-u_{i}\right)^{2}}{2 \lambda_{i}}-\frac{I^{\frac{2}{\delta}}}{R T}\right)$. Moreover, $(C)+(D 3)=0$ simplifies to $(C)=0$, since the equality of relative and internal temperatures implies $(D 3)=0$. This also implies $(C)=$ $\frac{3}{\tau}(1-(1-\theta) \nu)\left(1-R T \frac{1}{3} \sum_{i} \frac{1}{\lambda_{i}}\right)$. Consequently, $(C)=0$ implies $\frac{1}{3} \sum_{i} \frac{1}{\lambda_{i}}=\frac{1}{R T}$ : this means that Jensen inequality (24) is in fact an equality, and hence $\lambda_{1}=\lambda_{2}=\lambda_{3}=R T$.

Finally, we have obtained $f=\alpha \exp \left(-\frac{|v-u|^{2}}{2 R T}-\frac{I^{\frac{2}{\delta}}}{R T}\right)$, and hence $f=M_{p}(f)$, which concludes the proof.

\subsection{Equation on internal energy}

While the formulation of our model with the internal energy parameter $I$ is useful to prove the H-theorem, using the internal energy $\varepsilon(I)=I^{2 / \delta}$ turns out to be more convenient for numerical simulations (see section 5.1). If we denote by $g(t, x, v, \varepsilon)$ the corresponding distribution function (that is to say, such that $g d \varepsilon=f d I$ ), we get the following equation

$$
\partial_{t} g+v \cdot \nabla_{x} g=\mathcal{D}(g)
$$

where the collision operator is defined by

$$
\mathcal{D}(g)=\frac{1}{\tau}\left(\nabla_{v} \cdot\left((v-u) g+\Pi \nabla_{v} g\right)+\nabla_{\varepsilon}\left(2\left(\varepsilon-\frac{\delta}{2} R T_{r e l}\right) g+\nabla_{\varepsilon} 2\left(R T_{r e l} \varepsilon g\right)\right)\right) .
$$

The equilibrium and its Gaussian extension are now

$$
\mathcal{M}(g)=\frac{\rho \Lambda_{g}}{(2 \pi)^{3 / 2}} \frac{\varepsilon^{\frac{\delta-2}{2}}}{(R T)^{\frac{\delta}{2}}} \exp \left(-\frac{\frac{1}{2}(v-u)^{2}+\varepsilon}{R T}\right)
$$

and

$$
\mathcal{G}(g)=\frac{\rho \Lambda_{g}}{\sqrt{\operatorname{det}(2 \pi \Pi)}} \frac{\varepsilon^{\frac{\delta-2}{2}}}{\left(R T_{r e l}\right)^{\frac{\delta}{2}}} \exp \left(-\frac{1}{2}(v-u)^{t} \Pi^{-1}(v-u)-\frac{\varepsilon}{R T_{r e l}}\right)
$$

with

$$
\Lambda_{g}=\left(\int_{\mathbb{R}} \varepsilon^{\frac{\delta-2}{2}} \exp (-\varepsilon) d \varepsilon\right)^{-1} .
$$

When one deals with diatomic gases $(\delta=2)$ one recovers the same equation as before since $\varepsilon(I)$ is equal to $I$. The main difference with the $\varepsilon$-formulation of the problem is that the entropy inequality is transformed into a relative entropy inequality, that is

$$
\left\langle D_{g}(g) \log \left(\frac{g}{\varepsilon^{\frac{\delta-2}{2}}}\right)\right\rangle \leq 0,
$$

due to the change of variables. 


\section{Chapman-Enskog analysis}

From (12) and (23), it can easily be shown that the moments of $f$ satisfy the following conservation laws

$$
\begin{aligned}
& \partial_{t} \rho+\nabla_{x} \cdot \rho u=0, \\
& \partial_{t} \rho u+\nabla_{x} \cdot(\rho u \otimes u)+\nabla_{x} \cdot \Sigma(f)=0, \\
& \partial_{t} E+\nabla_{x} \cdot(E u+\Sigma(f) u+q(f))=0,
\end{aligned}
$$

where $\Sigma(f)$ and $q(f)$ denote the stress tensor and the heat flux, defined by

$$
\Sigma(f)=\langle(v-u) \otimes(v-u) f\rangle \quad q(f)=\left\langle\left(\frac{1}{2}|v-u|^{2}+I^{2 / \delta}\right)(v-u) f\right\rangle .
$$

The Chapman-Enskog procedure consists in looking for an approximation of $\Sigma(f)$ and $q(f)$ up to second order with respect to the Knudsen number $\varepsilon$, which is defined below, as functions of $\rho, u, T$ and their gradients.

To do so, we now write our model in a non-dimensional form. Assume we have some reference values of length $x_{*}$, pressure $p_{*}$, and temperature $T_{*}$. With these reference values, we can derive reference values for all the other quantities: mass density $\rho_{*}=p_{*} / R T_{*}$, velocity $v_{*}=\sqrt{R T_{*}}$, time $t_{*}=x_{*} / v_{*}$, distribution function $f_{*}=\rho_{*} /\left(R T_{*}\right)^{3 / 2}$. We also assume we have a reference value for the relaxation time $\tau_{*}$. By using the non-dimensional variables $w^{\prime}=w / w_{*}$ (where $w$ stands for any variables of the problem), our model can be written

$$
\partial_{t} f+v \cdot \nabla_{x} f=\frac{1}{\varepsilon} D(f)
$$

where $\varepsilon=\frac{v_{*} \tau_{*}}{x_{*}}$ is the Knudsen number. Note that since we always work with the non-dimensional variables from now on, these variables are not written with the' in (34).

Note that an important consequence of the use of these non-dimensional variables is that $R T$ has to be replaced by $T$ in every expressions given before. Namely, now $\Pi$ is defined by

$$
\Pi=(1-\theta)\left((1-\nu) T_{t r} I d+\nu \Theta\right)+\theta T I d
$$

the temperatures are now defined by

$$
E_{t r}=\frac{3}{2} \rho T_{t r}, \quad E_{\text {int }}=\frac{\delta}{2} \rho T_{\text {int }}, \quad \text { and } \quad E=\frac{1}{2} \rho|u|^{2}+\frac{3+\delta}{2} \rho T,
$$

and the Maxwellian of $f$ now is

$$
M_{p}(f)=\frac{\rho \Lambda_{\delta}}{(2 \pi)^{3 / 2}(T)^{(3+\delta) / 2}} \exp \left(-\frac{|v-u|^{2}}{2 T}-\frac{I^{2 / \delta}}{T}\right) .
$$

Now, it is standard to look for the deviation of $f$ from its own local equilibrium, that is to say to set $f=M_{p}(f)(1+\varepsilon g)$. However, this requires the linearization of the collision operator $D$ around $M_{p}(f)$, which is not very easy. At the contrary, it will be shown that it is much simpler to look for the deviation of $f$ from the anisotropic Gaussian $G_{p}(f)$ defined in (17). Since it can easily be seen that $M_{p}(f)$ and $G_{p}(f)$ are close up to $O(\varepsilon)$ terms, this expansion is sufficient to get the Navier-Stokes equations. We shall then prove the following proposition: 
Proposition 3.1. The moments of the solution of the kinetic model (34) satisfy, up to $O\left(\varepsilon^{2}\right)$, the Navier-Stokes equations

$$
\begin{aligned}
& \partial_{t} \rho+\nabla \cdot \rho u=0, \\
& \partial_{t} \rho u+\nabla \cdot(\rho u \otimes u)+\nabla p=-\nabla \cdot \sigma, \\
& \partial_{t} E+\nabla \cdot(E+p) u=-\nabla \cdot q-\nabla \cdot(\sigma u),
\end{aligned}
$$

where the shear stress tensor and the heat flux are given by

$$
\sigma=-\mu\left(\nabla u+(\nabla u)^{T}-\alpha \nabla \cdot u\right), \quad \text { and } \quad q=-\kappa \nabla \cdot T
$$

with the following values of the viscosity and heat transfer coefficients (in dimensional variables)

$$
\mu=\frac{\tau p}{2(1-(1-\theta) \nu)}, \quad \alpha=(\gamma-1)-\frac{(1-\nu)(1-\theta)}{\theta}\left(\frac{5}{3}-\gamma\right) \quad \text { and } \quad \kappa=\frac{5+\delta}{6} \tau p R,
$$

and $\gamma=\frac{\delta+5}{\delta+3}$. Moreover, the corresponding Prandtl number is

$$
\operatorname{Pr}=\frac{3}{2(1-(1-\theta) \nu)}
$$

In the first steps of the proof, we state some approximation properties.

Proposition 3.2. We write $f$ as $f=G_{p}(f)(1+\varepsilon g)$. Then we have:

$$
\left\langle\left(1, v, \frac{1}{2}|v|^{2}+I^{\frac{2}{\delta}}\right) G_{p}(f) g\right\rangle=0 .
$$

Moreover, if we assume that the deviation $g$ is an $O(1)$ with respect to $\varepsilon$, then we have

$$
\Pi=T I d+O(\varepsilon), \quad T_{t r}=T+O(\varepsilon), \quad T_{\text {int }}=T+O(\varepsilon), \quad T_{r e l}=T+O(\varepsilon) .
$$

and

$$
G_{p}(f)=M_{p}(f)+O(\varepsilon) .
$$

Proof. We have already noticed that $\left\langle\left(1, v, \frac{1}{2}|v|^{2}\right) G_{p}(f)\right\rangle=\left\langle\left(1, v, \frac{1}{2}|v|^{2}+I^{\frac{2}{\delta}}\right) f\right\rangle$ (see proposition 2.3), which gives (41). Relations (42) and (43) are obtained by simple Taylor expansions of (35) and (17) with respect to $\varepsilon$.

Now, we show that $\Sigma(f)$ and $q(f)$ can be approximated up to $O\left(\varepsilon^{2}\right)$ by using the previous decomposition.

Proposition 3.3. We have

$$
\begin{aligned}
& \Sigma(f)=p I d+\frac{\varepsilon}{1-(1-\theta) \nu} \Sigma\left(M_{p}(f) g\right)+\rho \frac{(1-\nu)(1-\theta)\left(T_{t r}-T\right)}{1-(1-\theta) \nu} I d+O\left(\varepsilon^{2}\right), \\
& \theta \rho\left(T_{t r}-T\right)=\frac{1}{3} \varepsilon \operatorname{trace}\left(\Sigma\left(M_{p}(f) g\right)\right)+O\left(\varepsilon^{2}\right) \\
& q(f)=\varepsilon q\left(M_{p}(f) g\right)+O\left(\varepsilon^{2}\right) .
\end{aligned}
$$


Proof. By using $f=G_{p}(f)(1+\varepsilon g)$, we have

$$
\Sigma(f)=\Sigma\left(G_{p}(f)\right)+\varepsilon \Sigma\left(G_{p}(f) g\right)=\rho \Pi+\varepsilon \Sigma\left(G_{p}(f) g\right) .
$$

But since by definition $\Sigma(f)=\rho \Theta(f),(35)$ also implies:

$$
\rho \Pi=(1-\theta)\left((1-\nu) \rho T_{t r} I d+\nu \Sigma(f)\right)+\theta \rho T I d .
$$

Using this relation in (47) gives $\Sigma(f)=(1-\theta)\left((1-\nu) \rho T_{t r} I d+\nu \Sigma(f)\right)+\theta \rho T I d+\varepsilon \Sigma\left(G_{p}(f) g\right)$, which, with (43), yields (44). Taking the trace of the previous relation gives (45). The approximation (46) is deduced from the decomposition $f=G_{p}(f)(1+\varepsilon g)$, the even parity of $G_{p}(f)$ with respect to $v$, and relation (43).

Consequently, the Navier-Stokes equations can be obtained provided that $\Sigma\left(M_{p}(f) g\right)$ and $q\left(M_{p}(f) g\right)$ can be approximated, up to $O(\varepsilon)$. This can be done by looking for an approximation of the deviation $g$ itself: it is obtained by using the decomposition $f=G_{p}(f)(1+\varepsilon g)$ into (34), which gives

$$
\partial_{t} G_{p}(f)+v \cdot \nabla_{x} G_{p}(f)+O(\varepsilon)=\frac{1}{\varepsilon} D\left(G_{p}(f)(1+\varepsilon g)\right) .
$$

First, we state what the expansion of $D\left(G_{p}(f)(1+\varepsilon g)\right)$ is.

Proposition 3.4. We have

$$
D\left(G_{p}(f)(1+\varepsilon g)\right)=\varepsilon \frac{1}{\tau} M_{p}(f) L g+O\left(\varepsilon^{2}\right),
$$

where $L$ is the linear operator

$$
L g=\frac{1}{M_{p}(f)}\left(\nabla_{v} \cdot\left(T M_{p}(f) \nabla_{v} g\right)+\partial_{I}\left(\frac{\delta^{2}}{2} T I^{2-\frac{2}{\delta}} M_{p}(f) \partial_{I} g\right)\right) .
$$

Proof.

Formulation (19) of $D(f)$ gives

$$
D\left(G_{p}(f)(1+\varepsilon g)\right)=\frac{1}{\tau} \nabla_{v, I} \cdot\left(\Omega G_{p}(f) \nabla_{v, I} \frac{G_{p}(f)(1+\varepsilon g)}{G_{p}(f)}\right) .
$$

A direct computation gives $\nabla_{v, I} \frac{G_{p}(f)(1+\varepsilon g)}{G_{p}(f)}=\varepsilon \nabla_{v, I} g$. Using this relation and the definition (16) of $\Omega$ gives:

$$
D\left(G_{p}(f)(1+\varepsilon g)\right)=\varepsilon \frac{1}{\tau}\left(\nabla_{v} \cdot\left(\Pi G_{p}(f) \nabla_{v} g\right)+\partial_{I}\left(\frac{\delta^{2}}{2} T_{r e l} I^{2-\frac{2}{\delta}} G_{p}(f) \partial_{I} g\right)\right) .
$$

Then the final result is obtained by using relations (42) and (43) to approximate $\Pi, G_{p}$, and $T_{r e l}$ up to $O(\varepsilon)$ by $T I d, M_{p}(f)$ and $T$, respectively. 
This proposition shows us that $g$ satisfies $M_{p}(f) L g=\tau\left(\partial_{t} G_{p}(f)+v \cdot G_{p}(f)\right)+O(\varepsilon)$, and then using (43) gives

$$
L g=\frac{\tau}{M_{p}(f)}\left(\partial_{t} M_{p}(f)+v \cdot \nabla_{x} M_{p}(f)\right)+O(\varepsilon)
$$

We now have to solve this equation approximately to obtain an approximation of $g$.

First, the right-hand side of (50) is expanded, and the time derivatives of $\rho, u$, and $T$ are approximated up to $O(\varepsilon)$ by their space gradients by using (32), (44), and (46). Then we get

$$
\frac{1}{M_{p}(f)}\left(\partial_{t} M_{p}(f)+v \cdot \nabla_{x} M_{p}(f)\right)=A(V, J) \cdot \frac{\nabla T}{\sqrt{T}}+B(V, J): \nabla u+O(\varepsilon)
$$

where $V=(v-u) / \sqrt{T}, J=\frac{I}{T^{\delta / 2}}$ and

$$
A(V, J)=\left(\frac{|V|^{2}}{2}+J^{2 / \delta}-\frac{5+\delta}{2}\right) V \quad \text { and } \quad B(V, J)=V \otimes V-\left(\frac{|V|^{2}+2 J^{2 / \delta}}{3+\delta}\right) I d .
$$

Then (50) reads

$$
L g=\tau\left(A(V, J) \cdot \frac{\nabla T}{\sqrt{T}}+B(V, J): \nabla u\right)+O(\varepsilon) .
$$

This equation can be solved by showing that $A$ and $B$ are eigenvectors of $L$. Indeed, we have the following proposition.

Proposition 3.5. The components of $A$ and $B$ satisfy

$$
L A_{i}=-3 A_{i} \quad \text { and } \quad L B_{i, j}=-2 B_{i, j} .
$$

Proof. By using the change of variables $V=(v-u) / \sqrt{T}$ and $J=\frac{I}{T^{\delta / 2}}$, the definition (48) reduces to

$$
L g=\frac{1}{M_{0}} \nabla_{V} \cdot\left(M_{0}(V, J) \nabla_{V} g\right)+\frac{1}{M_{0}} \partial_{J}\left(M_{0}(V, J) \frac{\delta^{2}}{2} J^{2-\frac{2}{\delta}} \partial_{J} g\right)+O(\varepsilon),
$$

where $M_{0}(V, J)=\frac{\Lambda_{\delta}}{(2 \pi)^{\frac{3}{2}}} \exp \left(-\frac{|V|^{2}}{2}-J^{2 / \delta}\right)$. The direct computation of $L A_{i}$ and $L B_{i, j}$ is easily obtained from (52) and is left to the reader.

This property shows that we can look for an approximate solution of (51) as a linear combination of the components of $A$ and $B$. We find

$$
g=-\frac{\tau}{3} \frac{\nabla T}{\sqrt{T}} \cdot A(V, J)-\frac{\tau}{2} \nabla u: B(V, J)+O(\varepsilon) .
$$

Then we just have to insert this expression into $\Sigma\left(M_{p}(f) g\right)$ and $q\left(M_{p}(f) g\right)$ to get, after calculation of Gaussian integrals,

$$
\begin{aligned}
& \Sigma\left(M_{p}(f) g\right)=-\frac{\tau p}{2}\left(\nabla u+(\nabla u)^{T}-(\gamma-1)(\nabla \cdot u) I d\right)+O(\varepsilon), \\
& q\left(M_{p}(f) g\right)=-\frac{\tau p(5+\delta)}{6} \nabla T+O(\varepsilon) .
\end{aligned}
$$


Now we use proposition 3.3 and we come back to the dimensional variables to get

$$
\Sigma(f)=p I-\mu\left(\nabla u+(\nabla u)^{T}-\alpha \nabla \cdot u\right), \quad \text { and } \quad q=-\kappa \nabla \cdot T,
$$

up to $O\left(\varepsilon^{2}\right)$, with the following values of the viscosity and heat transfer coefficients

$$
\mu=\frac{\tau p}{2(1-(1-\theta) \nu)}, \quad \alpha=(\gamma-1)-\frac{(1-\nu)(1-\theta)}{\theta}\left(\frac{5}{3}-\gamma\right), \quad \text { and } \quad \kappa=\frac{5+\delta}{6} \tau p R .
$$

Using these relations into the conservation laws (32) proves that $\rho, \rho u$, and $E$ solve the NavierStokes equations (37) up to $O\left(\varepsilon^{2}\right)$ with transport coefficients given by (39) and that the Prandtl number is

$$
\operatorname{Pr}=\frac{\mu}{\kappa} \frac{(5+\delta) R}{2}=\frac{3}{2(1-(1-\theta) \nu)} .
$$

\section{The ES-Fokker Planck model with a non constant $\nu$}

This short section shows how we adjust the Prandtl number in all cases, following a procedure proposed for monoatomic gases [17].

\subsection{Some limits of the model with a constant $\nu$}

In the previous section, we have found that the Prandtl number obtained for the polyatomic ESFokker Planck model is

$$
\operatorname{Pr}=\frac{\mu}{\kappa} \frac{(5+\delta) R}{2}=\frac{3}{2(1-(1-\theta) \nu)},
$$

and can be adjusted to various values by choosing a corresponding value of the parameter $\nu$.

Moreover, we have seen that the model is well defined (that is to say that the tensor $\Pi$ is positive definite for every $f$ ) if, and only if, $-\frac{1}{2}<\nu<1$. This last condition leads to the following limitations for the Prandtl number:

$$
1<\operatorname{Pr}<+\infty
$$

However, the correct Prandtl number for polyatomic gases, which depends on $\delta$, is smaller than 1 , and hence cannot be obtained with our model.

In the next section, we show that this analysis, which is based on the inequality (22) is too restrictive, and that there is a simple way to adjust the correct Prandtl number.

\subsection{Recovering the correct Prandtl number}

\subsubsection{General case}

The previous analysis relies on inequality (22) of proposition 2.4 that does not take into account the distribution $f$ itself: this inequality ensures the positive definiteness of $\Pi$ independently of $f$. However, proposition 2.4 also indicates that $\Pi$ is positive definite for a given $f$ if $\nu$ satisfies (21). This inequality is less restrictive, since it depends on $f$ via the temperature $T$ and the extremal eigenvalues of $\Theta$. 
Consequently, we take $\nu$ in the larger interval $\left[-\frac{R T_{t r}+\frac{\theta}{1-\theta} R T}{\lambda_{\max }-R T_{t r}}, 1\right]$. This means that $\nu$ is a non constant value, since $T, T_{t r}$, and $\lambda_{\max }$ depend on time and space. This allows us to hope for a Prandtl number smaller than 1, while the proposition 2.5 ensures that the model is well defined.

However, it is not obvious that this interval is large enough to adjust any small value of the Prandtl number. Our idea is that the Prandtl number makes sense when the flow is close to the equilibrium, that is to say when $f$ is close to its own local Maxwellian $M_{p}(f)$. In such case, $\Theta=R T I+O(\varepsilon)$, and all the eigenvalues of $\Theta$ are close to $R T$ up to $O(\varepsilon)$. This implies $\lambda_{\max }=$ $R T(1+C \varepsilon)$ and $T_{t r}=T(1+D \varepsilon)$, and since by definition $\lambda_{\max }>R T_{t r}$, then $C>D$. Consequently, the smallest possible value of $\nu$ behaves like $-\frac{1}{(1-\theta)(C-D) \varepsilon}$ which can be as small as possible for small $\varepsilon$. This shows that $\nu$ can take any arbitrary value between $-\infty$ and 1 when $\varepsilon$ is small enough, and hence the Prandtl number can be made arbitrarily small.

\subsubsection{Case of a diatomic gas: air}

For air, the Prandtl number is estimated to $\operatorname{Pr} \approx 0.71$. Since $\theta=0.2$ is generally used in literature [1] for air, one gets that we need to use $\nu_{\text {air }} \approx-1.3908$.

In other words, by defining $\nu$ as a non constant value that satisfies (21) and is lower than 1 , we can adjust the correct Prandtl number provided that $\nu$ can be set to $\nu_{\text {air }}$ near the equilibrium regime.

This analysis suggests a very simple definition of $\nu$ : we propose to use the smallest negative $\nu$ such that:

- $\Pi$ remains strictly definite positive,

- $\nu \geq \nu_{\text {air }}$

This leads to the following definition:

$$
\nu=\max \left(\nu_{a i r},-\frac{R T_{t r}+\frac{\theta}{1-\theta} R T}{\lambda_{\max }-R T_{t r}}\right) .
$$

Note that in most realistic cases $\nu$ is equal to $\nu_{\text {air }}$. Indeed, $\nu \neq \nu_{\text {air }}$ implies $\lambda_{\text {max }}>1.82 R T_{t r}$ for $\theta=0.2$ because $T=\frac{3}{5} T_{t r}+\frac{2}{5} T_{\text {int }} \geq \frac{3}{5} T_{t r}$, which can only happen in case of a highly non equilibrium flow with strong directional non isotropy: such a case is very specific and is usually not observed in aerodynamical flows, for instance.

\section{$5 \quad$ Numerical tests}

Due to the diffusion operator used in our model, we find it attractive to use a stochastic simulation method (like in [13], see also [15]). In this paper, our study is restricted to space homogeneous problems, since this is sufficient to illustrate the main property of our model. Further studies will be done in inhomogeneous cases and comparisons with full Boltzmann equation and/or ESBGK model will be provided in a forthcoming paper. In this section, we use our model written in energy variable (see section 2.3):

$$
\partial_{t} g=\frac{1}{\tau}\left(\nabla_{v} \cdot\left((v-u) g+\Pi \nabla_{v} g\right)+\partial_{\varepsilon}\left(2\left(\varepsilon-\frac{\delta}{2} R T_{r e l}\right) g+\partial_{\varepsilon} 2\left(R T_{r e l} \varepsilon g\right)\right)\right) .
$$


Note that here, we use dimensional variables only. Consequently, the parameter $\varepsilon$ is here the molecular internal energy, and should not be confused with the Knudsen number used in section 3.

Our goal is to show numerically that we are able to capture the correct Prandtl number for polyatomic gases. This numerical illustration is based on the following remarks. First, the density, velocity, and temperature of $f$ are constant in time: this is due to the conservation properties of the collision operator. Second, the heat flux $q$ defined for $g$ as $q:=\left\langle\left(\frac{1}{2}|v-u|^{2}+\varepsilon\right)(v-u) g\right\rangle$ satisfies

$$
\frac{d}{d t} q=-\frac{3}{\tau} q
$$

so that

$$
q(t)=q(0) \exp \left(-\frac{3 t}{\tau}\right) .
$$

Finally, the tensor $\Theta$ satisfies the relaxation equation

$$
\begin{aligned}
\frac{d}{d t} \Theta & =\frac{2}{\tau}(\Pi-\Theta) \\
& =\frac{2}{\tau}(1-\theta)(1-\nu)\left(R T_{t r} I d-\Theta\right)+\frac{2}{\tau} \theta(R T I d-\Theta)
\end{aligned}
$$

and its trace satisfies

$$
\frac{d}{d t} 3 R T_{t r}=\frac{2}{\tau} \theta\left(3 R T-3 R T_{t r}\right)
$$

which shows that $\Theta$ tends to RTI for large times, and hence $\nu$ tends to the constant value $\nu_{\text {air }}$ (see (54)). Then for large times $t>s, \nu$ can be assumed to be constant, and (58) can be solved to give

$$
\Theta(t)-R T_{t r}(t) I d=\exp \left(-\frac{2((1-\nu)(1-\theta)+\theta)(t-s)}{\tau}\right)\left(\Theta(s)-R T_{t r}(s) I d\right) .
$$

Using equations (60) and (57), one gets

$$
\frac{\log \left(\left|\Theta_{i, i}(t)-R T_{t r}(t)\right| /\left|\Theta_{i, i}(s)-R T_{t r}(s)\right|\right)}{\log \left(\left|q_{i}(t)\right| /\left|q_{i}(s)\right|\right)}=\frac{2(1-(1-\theta) \nu)}{3}=\frac{1}{\operatorname{Pr}}
$$

for $i=1,2,3$, so that we are able to recover the Prandtl number by looking at the long time values of $\Theta(t)$ and $q(t)$.

\subsection{A stochastic numerical method}

The distribution function is approximated with $N$ numerical particles so that

$$
f(t, v) \simeq \alpha \sum_{i}^{N} \omega_{i} \delta_{V_{i}(t), \varepsilon_{i}(t)},
$$

where $\omega_{i}$ is the numerical weight of numerical particle $i$ and $\delta_{V_{i}(t)}$ is the Dirac function at the particle velocity $V_{i}(t)$ and particle internal energy $\varepsilon_{i}(t)$. Moreover $\alpha$ is defined through the constant density $\rho=\int_{\mathbb{R}^{3}} f(t, v) d v$ by

$$
\rho=\alpha \sum_{i}^{N} \omega_{i} .
$$


In the test cases presented here, all numerical weights $\omega_{i}$ are equal, and we just have to define the dynamics of the numerical particles. To solve diffusive problems, it is well-known that using Brownian motion is a good way to proceed (see [15]): the corresponding stochastic ordinary differential equation is called the Ornstein-Uhlenbeck process that reads

$$
\begin{aligned}
& d V_{i}(t)=-\frac{d t}{\tau}\left(V_{i}(t)-u\right)+A_{v} d B_{v}(t) \\
& d \varepsilon_{i}(t)=-\frac{2 d t}{\tau}\left(\varepsilon_{i}(t)-\frac{\delta}{2} R T_{\text {rel }}\right)+2 \sqrt{R T_{r e l} \varepsilon_{i}(t)} d B_{\varepsilon}(t)
\end{aligned}
$$

for each $1 \leq i \leq N$. The quantities $d B_{v}(t)$ and $d B_{\varepsilon}(t)$ respectively are three dimensional and one dimensional Brownian processes. The matrix $A_{v}$ has to satisfy $A_{v} A_{v}^{T}=\Pi$. Several choices are available for $A_{v}$ : we find it simpler to use the Choleski decomposition. For the time discretization we use a backward Euler method for velocities. The complete algorithm for the velocity part of the algorithm is explained and validated in [17].

We now focus on the internal energy equation. Since the diffusion coefficient depends on the square root of the internal energy, this latter variable must remain positive, and hence a simple Euler algorithm cannot work in this case. We will adapt the Milshtein scheme (see [15]) that reads on our problem:

$$
\varepsilon_{i}^{n+1}=\left(\left(\sqrt{\varepsilon_{i}^{n}}+\sqrt{\frac{d t}{\tau} R T_{r e l}} B_{\varepsilon}\right)^{2}+(\delta-1) R T_{r e l} \frac{d t}{\tau}\right) /\left(1+\frac{2 d t}{\tau}\right),
$$

with $B_{\varepsilon}$ a random number chosen from a standard normal law.

The global scheme on velocities and internal energies preserves the mass but does not preserve the momentum and the energy, like most Monte-Carlo methods. However, these quantities are preserved in a statistical way. Moreover at the end of each time step, the distribution is renormalized to keep the mean velocity constant and to decrease the error in $\Theta$.

Now we focus on the validation of our numerical method. We do not re-validate the velocity scheme alone since its was done in [17]. We are going to validate the energy part which is new and also the global numerical scheme to see if we can recover the correct Prandtl number.

\subsection{Validation of the stochastic simulation of the internal energy equation}

First, we remind that in an homogeneous case, the temperature $T$ does not change and that the internal temperature varies according to the following law:

$$
\frac{d}{d t} T_{\text {int }}=-\frac{2}{\tau}\left(T_{\text {int }}-T_{\text {rel }}\right)=\frac{2}{\tau} \theta\left(T-T_{\text {int }}\right)
$$

so that the internal energy density is $E_{\text {int }}(t)=\frac{\delta}{2} R T_{\text {int }}(0) \exp \left(-2 \theta \frac{t}{\tau}\right)$.

We use 1 million particles for validation and the following parameters:

- the initial internal energies are defined by $1000^{2}(2 s+1)$ where $s$ follows a uniform law in $[0,1]$

- $\theta$ is set to 1 so that $T_{r e l}=T$, and $\frac{\delta}{2} R T$ is equal to 7.500 .000 ; 
- the initial value of $\frac{\delta}{2} R T_{\text {int }}$ is set to 2.500 .000 ;

- $\tau$ is chosen to be equal to 1 ;

- the final time is equal to 5 which is sufficient to reach the equilibrium;

- the time step is equal to 0.005 so that we do 1000 iterations.

We will test two values of $\delta$ to validate the model.

\subsubsection{Polyatomic case: $\delta=6$}

Using equation (65) we can compare the behavior of the first moment with the theoretical result. There is a very good agreement between our results and the theory: we recover the exponential convergence in time of the mean internal energy in figure 1 , and the relative error is of order $10^{-3}$ when we compare the analytical solution with the numerical solution of equation 65 in figure 2 . Moreover the comparison between the theoretical equilibrium which is $\Lambda_{g} \varepsilon^{\frac{\delta-2}{2}}(R T)^{-\frac{\delta}{2}} \exp (-\varepsilon / R T)$ is very good (figure 3 ).

\subsubsection{Diatomic case: $\delta=2$}

The conclusions are the same for $\delta=2$ :

- good agreement for exponential convergence on internal energy (figure 4),

- relative error is very small (figure 5),

- the correct equilibrium is obtained in figure 6 .

\subsection{Numerical results for the polyatomic model}

We now present two test cases to validate the ES Fokker-Planck for polyatomic gases. In the first one the flow is close to isotropic so that the $\nu$ correction (54) is not active, while in the second one it will be activated in during an initial transient phase.

\subsubsection{First test case: a diatomic gas}

In this test case, we use air whose Prandtl number is approximately 0.71. We take the standard value $\theta=0.2$, and the Prandtl number is adjusted with $\nu_{\text {air }}=-1.3908$. The initial data is not too anisotropic so that the correction on $\nu$ is not active.

We use 2 million particles. We choose three independent laws for the three components of velocity of the numerical particles:

- the first component is equal to $1000 s^{4}-200$ where $s$ follows a uniform law between $[0,1]$,

- the second component of velocity is equal to $1000 s-500$ where $s$ follows a uniform law between $[0,1]$,

- the second component of velocity is equal to $1000 s-500$ where $s$ follows a uniform law between $[0,1]$. 
The internal energies are defined by $40.000 s+40.000$ where $s$ follows a uniform law between $[0,1]$.

The timestep is set to 0.02 . The final simulation time is $10 \tau$, where the relaxation time is set to $\tau=1$ to catpure the relaxation towards equilibrium for velocities and internal energies (whose characteristic time is $5 \tau$ ).

On figure 7 we can observe after some time the convergence of the directional tranlational temperatures $T_{11}, T_{22}, T_{33}$ (diagonal components of $\Theta$ ). towards the same translational temperature $T_{t r}$, and later the convergence of all these temperatures with the internal energy temperature $T_{\text {int }}$ to the equilibrium temperature $T$.

At convergence we recover the Maxwellian distribution on velocities (figure 8) and the exponential one on internal energies (figure 9).

On figure 10 we observe the convergence of the heat flux $q$ and the quantity $T_{11}-T_{t r}$ towards zero. Due to the numerical noise induced by the random method, one has to consider only the beginning of the curves for a relevant comparison with the theory. Equation (57) shows that the profile of $\log (|q / q(0)|)$ should have a slope -3 since $\tau=1$. Numerically we obtain -3.0029 . For the temperature, equation (60) shows that the slope of $\log \left|T_{11}(t)-T_{t r}(t)\right| /\left|T_{11}(0)-T_{t r}(0)\right|$ should be $2(1-\nu(1-\theta))=4.2253$ : numerically, we obtain 4.2091 . Therefore the results are in very good agreement with the theory.

\subsubsection{Second test case}

In this second test case we want to test the correction (54) of $\nu$. To do so we only change the law of the first component of the velocity to $5000 s^{4}-1000$ where $s$ follows a uniform law in $[0,1]$, which induces now a very nonisotropic tensor $\Theta$.

We keep the same numerical parameters except for final time which is set to 1 , since after that $\nu$ is not corrected and we recover the previous test case. We still have a convergence of the temperatures as before (figure (11)): since we stop the computations at $t=1$ relaxation between internal energy and translationnal energy is still in progress. Nonetheless since our initial date is anisotropic in velocity, the correction on $\nu$ is activated so that the Prandtl is not correct (we are far from equilibrium...) as it can be seen in figure (12). As a consequence initially the log of the difference $\left(T_{11}-T_{t r}\right)$ is not a linear function of time. But as soon as $\nu$ is equal to $\nu_{a i r}$ we go back to the first test case and it works (figure 13).

\section{Conclusion}

In this paper, we have proposed an extension of the ES-Fokker-Planck model of the Boltzmann equation to polyatomic gases. This model satisfies the properties of conservation of the Boltzmann equation, and it has been defined so as to allow for correct transport coefficients. This has been illustrated by numerical tests for homogeneous problems. Moreover, it has been proved that this new model satisfies the H-theorem. An application of our model to non-homogeneous flows will be proposed in a future work.

Acknowledgments. This study has been carried out in the frame of "the Investments for the future" Programme IdEx Bordeaux - CPU (ANR-10-IDEX-03-02). 


\section{References}

[1] P. Andriès, P. Le Tallec, J.-F. Perlat, and B. Perthame. The Gaussian-BGK model of Boltzmann equation with small Prandtl number. Eur. J. Mech. B/Fluids, 2000.

[2] C. Baranger, J. Claudel, N. Hérouard, and L. Mieussens. Locally refined discrete velocity grids for stationary rarefied flow simulations. Journal of Computational Physics, 257, Part A(0):572 $-593,2014$.

[3] G.A. Bird. Molecular Gas Dynamics and the Direct Simulation of Gas Flows. Oxford Science Publications, 1994.

[4] S. Chen, K. Xu, C. Lee, and Q. Cai. A unified gas kinetic scheme with moving mesh and velocity space adaptation. Journal of Computational Physics, 231(20):6643 - 6664, 2012.

[5] S. Chigullapalli and A. Alexeenko. Unsteady 3d rarefied flow solver based on boltzmann-esbgk model kinetic equations. In 41st AIAA Fluid Dynamics Conference and Exhibit. AIAA, June 27-30 2011. AIAA Paper 2011-3993.

[6] D.H. Davis. Monte carlo calculation of molecular flow rates through a cylindrical elbow and pipes of other shapes. J. Appl. Phys., 31:1169-1176, 1960.

[7] L. Desvillettes, R. Monaco, and F. Salvarani. A kinetic model allowing to obtain the energy law of polytropic gases in the presence of chemical reactions. European Journal of Mechanics - B/Fluids, 24(2):219 - 236, 2005.

[8] Laurent Desvillettes. Sur un modèle de type borgnakke - larsen conduisant à des lois d'énergie non linéaires en température pour les gaz parfaits polyatomiques. Annales de la Faculté des sciences de Toulouse : Mathématiques, 6(2):257-262, 1997.

[9] G. Dimarco and L. Pareschi. Numerical methods for kinetic equations. Acta Numerica, 23:369$520,2014$.

[10] Hossein Gorji and Patrick Jenny. A Kinetic Model for Gas Mixtures Based on a Fokker-Planck Equation. Journal of Physics: Conference Series, 362(1):012042-, 2012.

[11] M. Hossein Gorji and Patrick Jenny. A Fokker-Planck based kinetic model for diatomic rarefied gas flows. Physics of fluids, 25(6):062002-, June 2013.

[12] M.H. Gorji, M. Torrilhon, and Patrick Jenny. Fokker-Planck model for computational studies of monatomic rarefied gas flows. Journal of fluid mechanics, 680:574-601, August 2011.

[13] Patrick Jenny, Manuel Torrilhon, and Stefan Heinz. A solution algorithm for the fluid dynamic equations based on a stochastic model for molecular motion. Journal of computational physics, 229(4):1077-1098, 2010.

[14] V.I. Kolobov, R.R. Arslanbekov, V.V. Aristov, A.A. Frolova, and S.A. Zabelok. Unified solver for rarefied and continuum flows with adaptive mesh and algorithm refinement. Journal of Computational Physics, 223(2):589 - 608, 2007. 
[15] Bernard Lapeyre, Étienne Pardoux, Rémi Sentis, and Société de mathématiques appliquées et industrielles (France) ). Méthodes de Monte-Carlo pour les équations de transport et de diffusion. Mathématiques et applications. Springer, Berlin, 1998. Cet ouvrage est une version remaniée des notes d'un cours présenté par les auteurs en préliminaire au 25e Congrès d'analyse numérique, les 22 et 23 mai 1993.

[16] Z.-H. Li and H.-X. Zhang. Gas-kinetic numerical studies of three-dimensional complex flows on spacecraft re-entry. Journal of Computational Physics, 228(4):1116 - 1138, 2009.

[17] J. Mathiaud and L. Mieussens. A Fokker-Planck model of the Boltzmann equation with correct Prandtl number. Journal of Statistical Physics, 162(2):397-414, 2016.

[18] L. Mieussens. A survey of deterministic solvers for rarefied flows. submitted, 2014.

[19] G.A. Radtke, N. G. Hadjiconstantinou, and W. Wagner. Low-noise monte carlo simulation of the variable hard-sphere gas. Physics of Fluids, 23:030606, 2011.

[20] V.A. Titarev. Efficient deterministic modelling of three-dimensional rarefied gas flows. Commun. Comput. Phys., 12(1):162-192, 2012.

[21] L. Wu, J.M. Reese, and Y.H. Zhang. Oscillatory rarefied gas flow inside rectangular cavities. Journal of Fluid Mechanics, 748:350-367, 2014. 


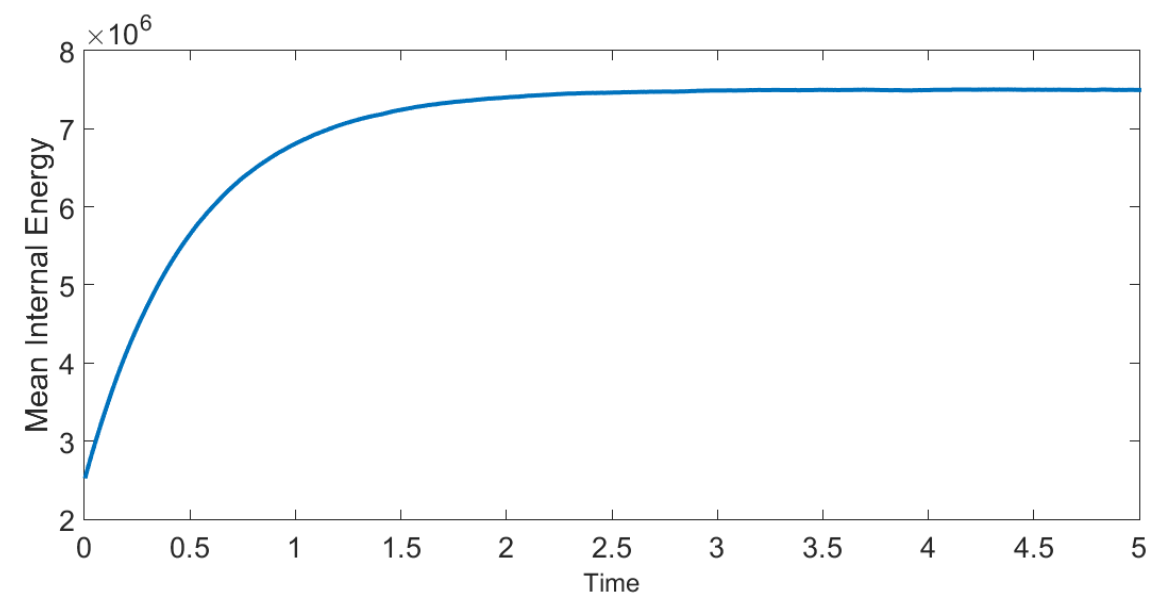

Figure 1: Time evolution of the mean internal energy $(\delta=6)$.

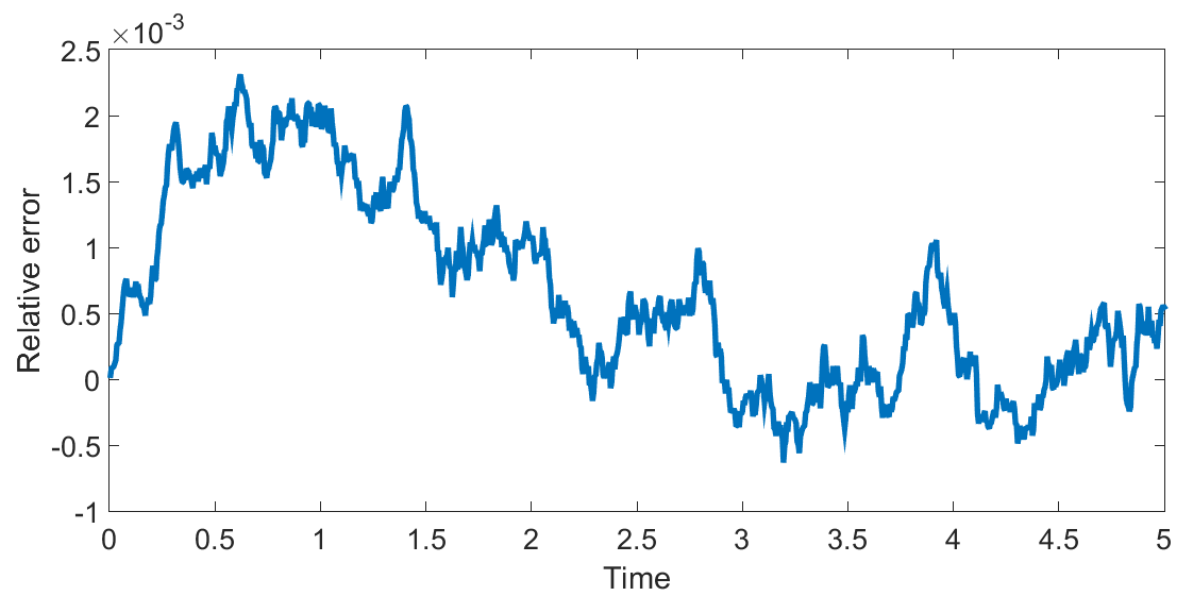

Figure 2: Time evolution of the relative error on the mean internal energy $(\delta=6)$. 


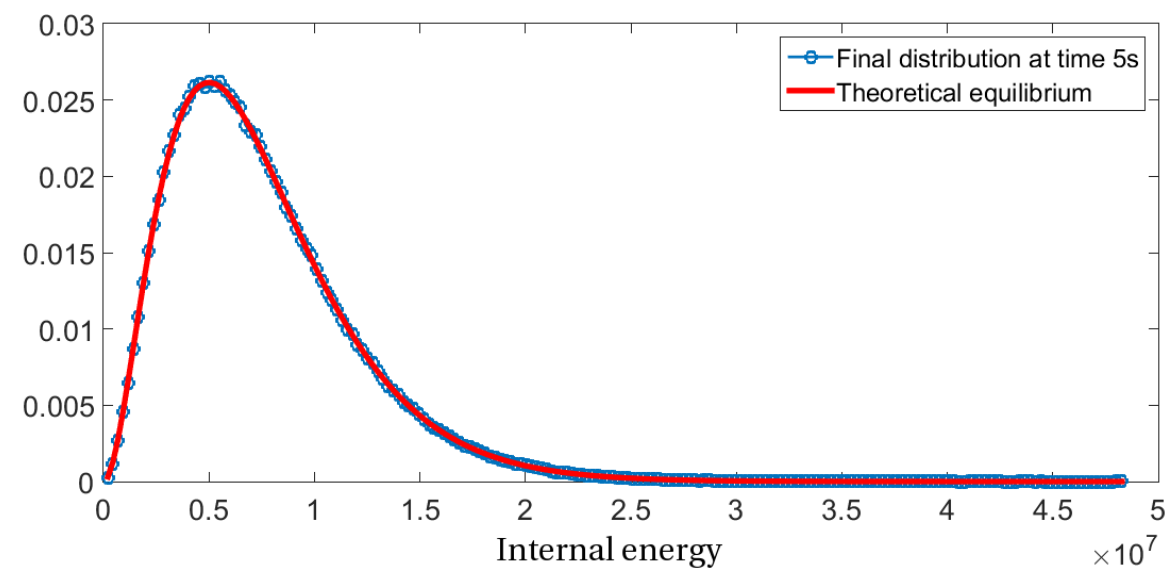

Figure 3: Theoretical internal energy distribution at equilibrium vs numerical distribution at final time $(\delta=6)$ : the equilibrium state is reached.

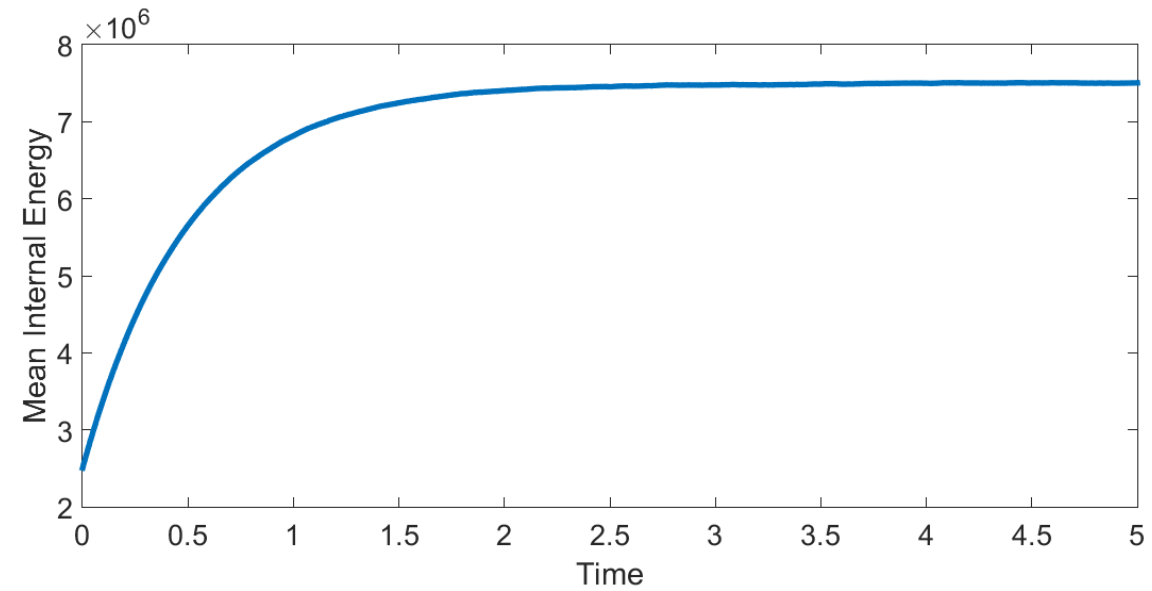

Figure 4: Time evolution of the mean internal energy $(\delta=2)$. 


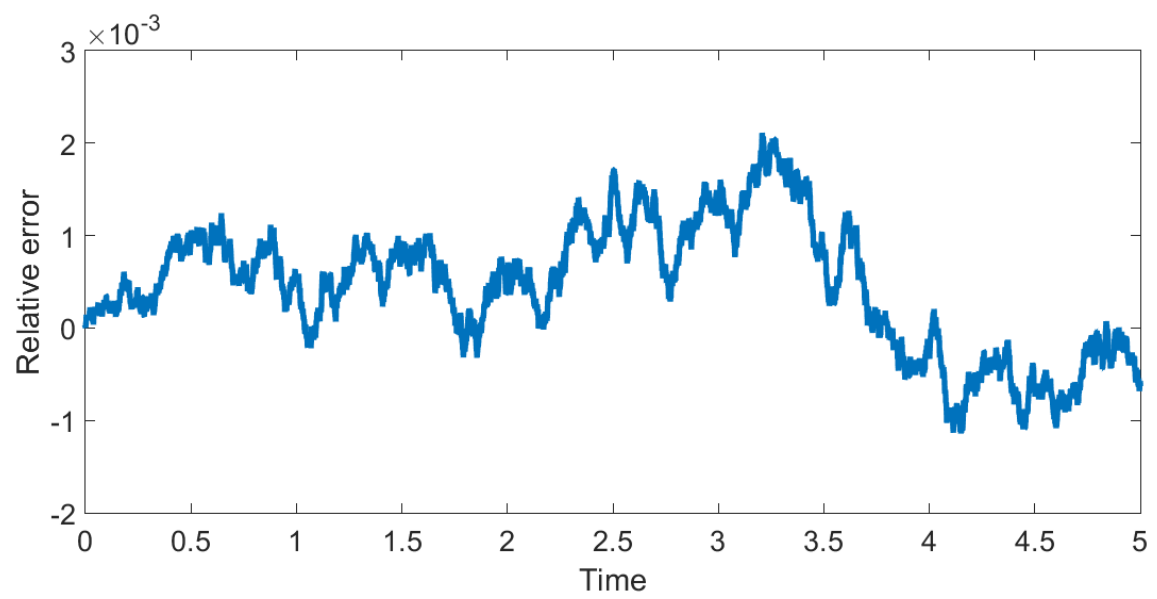

Figure 5: Time evolution of the relative error on the mean internal energy $(\delta=2)$.

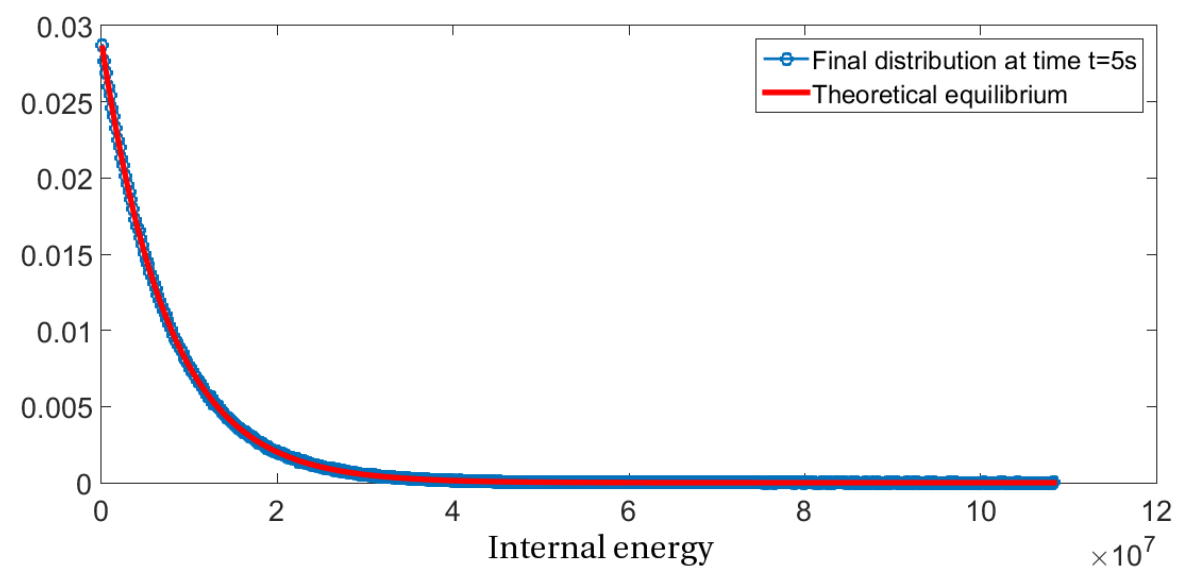

Figure 6: Theoretical equilibrium vs numerical equilibrium $(\delta=2)$. 


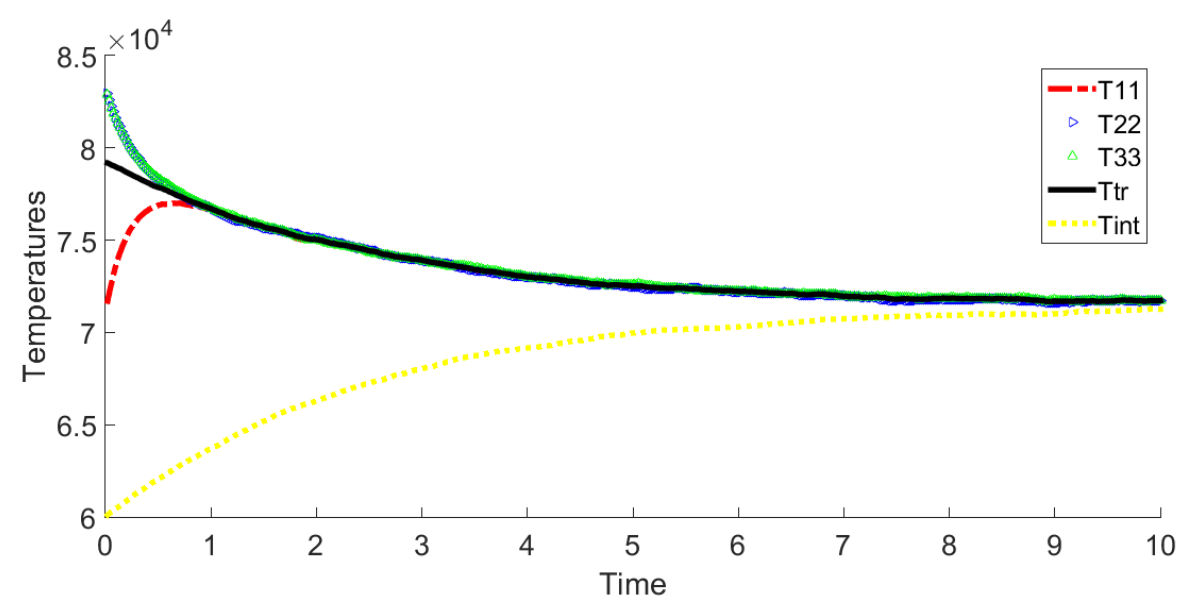

Figure 7: Convergence of the directional translational temperatures and the internal temperature to their equilibrium value.

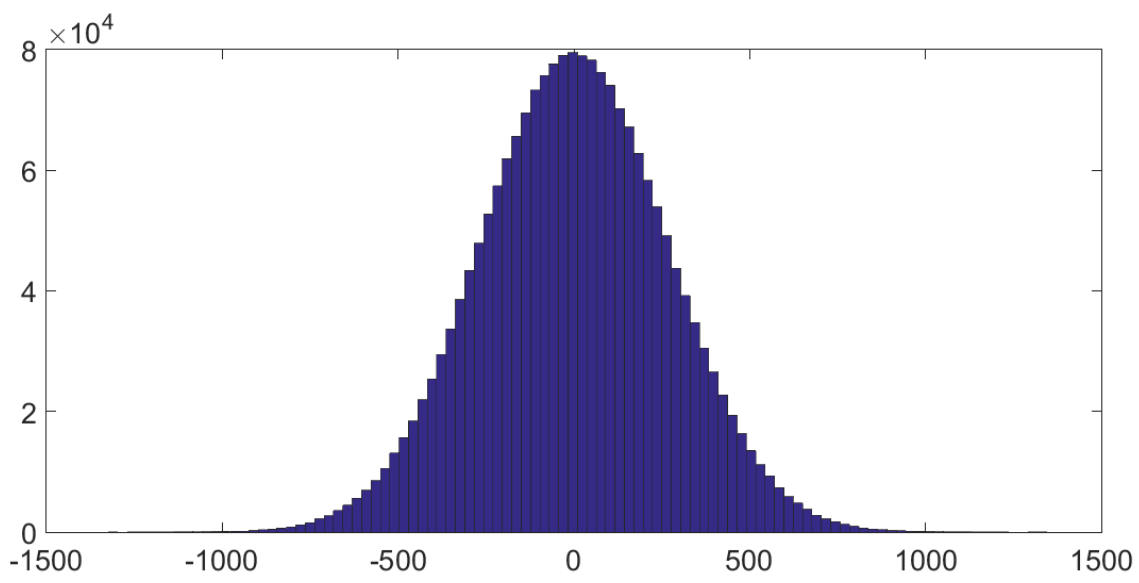

Figure 8: Maxwellian equilibrium for the distribution of the first component of the velocities, at time $t=10 \mathrm{~s}$ : number of numerical particles on the $y$ axis as a function of the $V_{x}$ component 


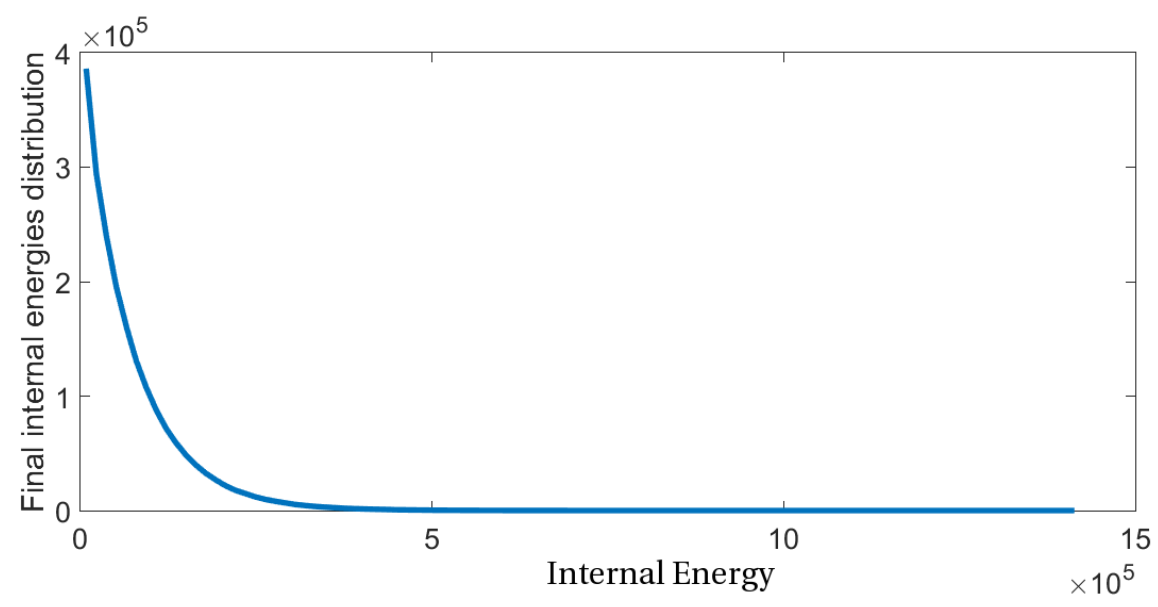

Figure 9: Distribution of the internal energies at time $t=10$ : exponential equilibrium.

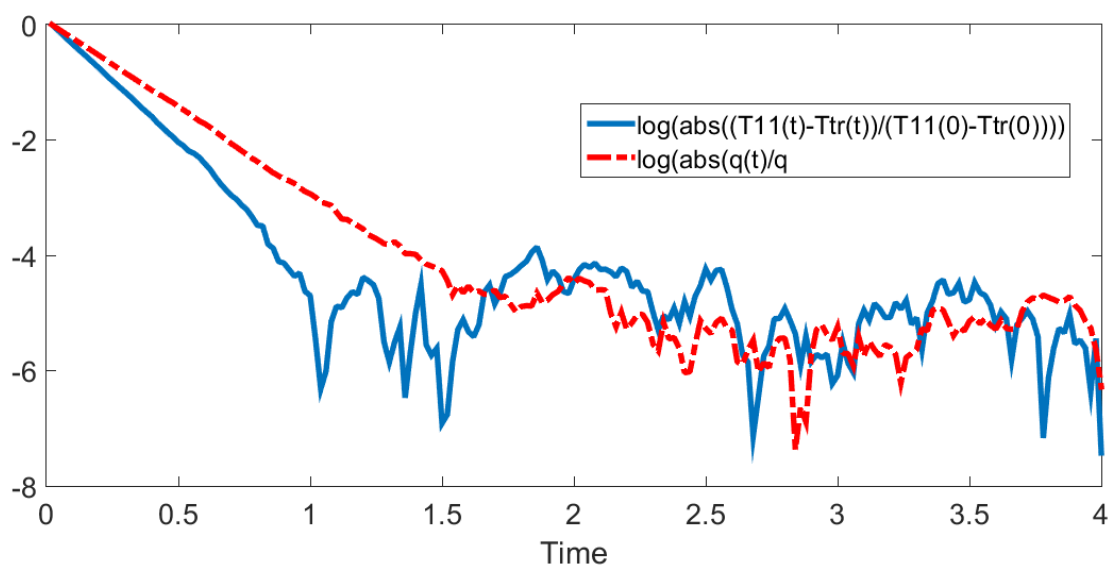

Figure 10: Convergence to the equilibrium of the heat flux and a directional temperature. 


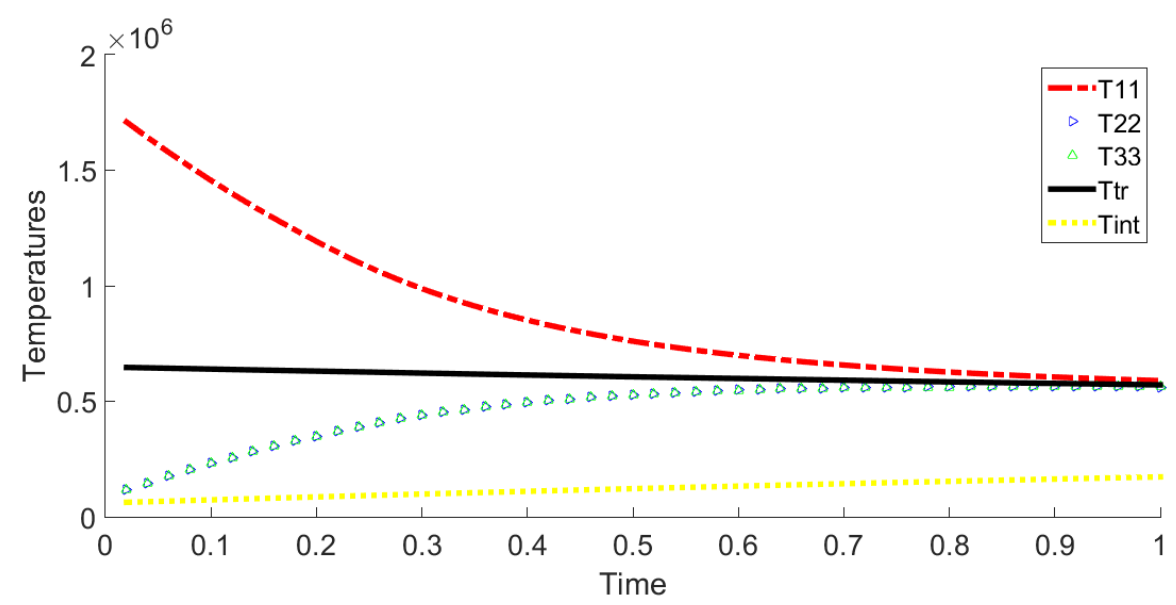

Figure 11: Convergence of the directional translational temperatures and the internal temperature to their equilibrium value.

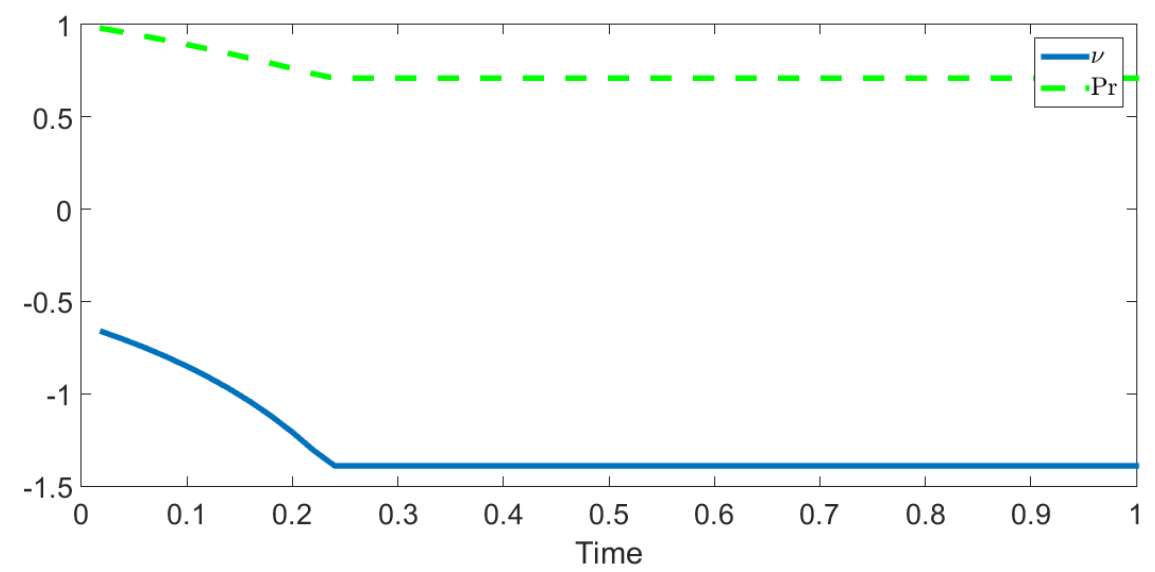

Figure 12: Time evolution of the parameter $\nu$ and of the numerical Prandtl number (estimated by $(61))$. 


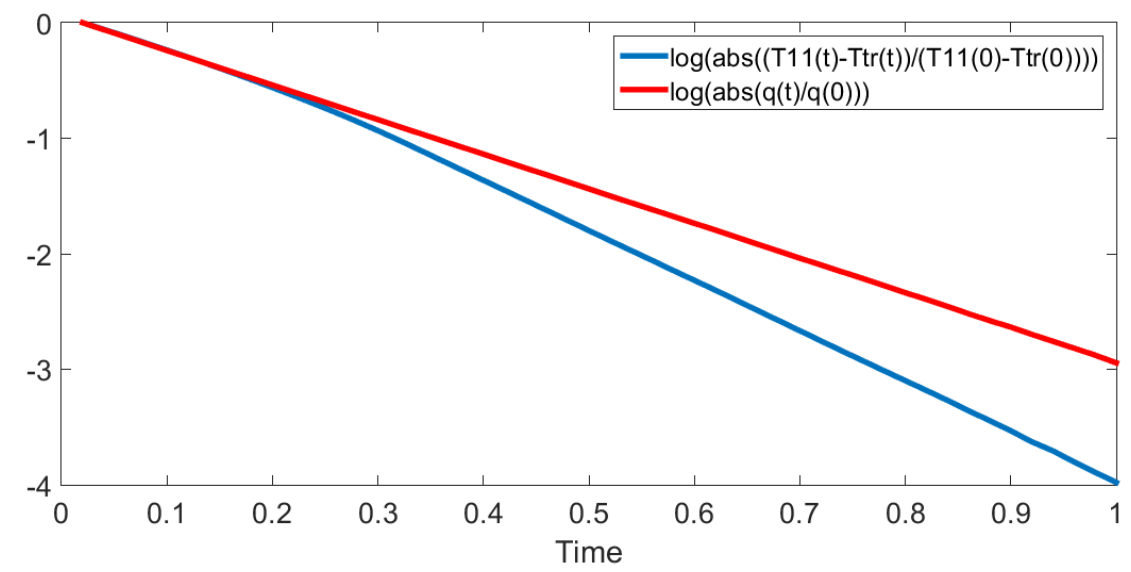

Figure 13: Convergence to the equilibrium of the heat flux and a directional temperature. 